\title{
Allergien beim Pferd - Stand der Wissenschaft
}

Interview mit Frau Prof. Bettina Wagner

\begin{abstract}
Allergisch bedingte Erkrankungen werden beim Pferd immer wieder vermutet. Aber sind alle diese Erkrankungen wirklich allergischer Natur? Welche Allergene kommen infrage? Und welche diagnostischen und therapeutischen Möglichkeiten stehen dem Tierarzt zur Verfügung? In einem Interview mit dem pferde.spiegel erläutert Frau Prof. Bettina Wagner (Associate Professor of Immunology, College of Veterinary Medicine, Cornell University) den aktuellen Stand der Wissenschaft zum Thema Allergien beim Pferd.
\end{abstract}

Frau Prof. Wagner, Allergien werden beim Pferd immer wieder vermutet. Welche Erkrankungen sind wirklich allergisch bedingt?

Beim Pferd gibt es mehrere Erkrankungen, die in der Literatur als mögliche Allergien diskutiert werden. Dennoch ist es nur für 2 Erkrankungen wirklich gezeigt, dass sie allergischer Natur sind: das Sommerekzem und die Urtikaria. Bei diesen beiden Erkrankungen sind wir sehr sicher, dass es sich um eine typische Typ-I-Allergie handelt, also eine Immunglobulin-E (IgE)-vermittelte Überempfindlichkeit des Immunsystems.

Haben alle anderen Erkrankungen dann keine allergische Ätiologie?

Es gibt verschiedene andere Erkrankungen, bei denen diskutiert wird, ob sie allergischer Natur sind. Eine ist z.B. die respiratorische Erkrankung, die in Deutschland immer noch gerne als COPD bezeichnet wird, und in der internationalen Literatur RAO (Recurrent Airway Obstruction) oder mittlerweile IAD (Inflammatory Airway Disease) genannt wird. Diese Erkrankung wurde für lange Zeit in der Literatur als Allergie zitiert und es wurde angenommen, dass IgE-Antikörper eine zentrale Rolle bei der Entstehung der Erkrankung spielen. Es gibt jedoch nur eine Studie, die vor

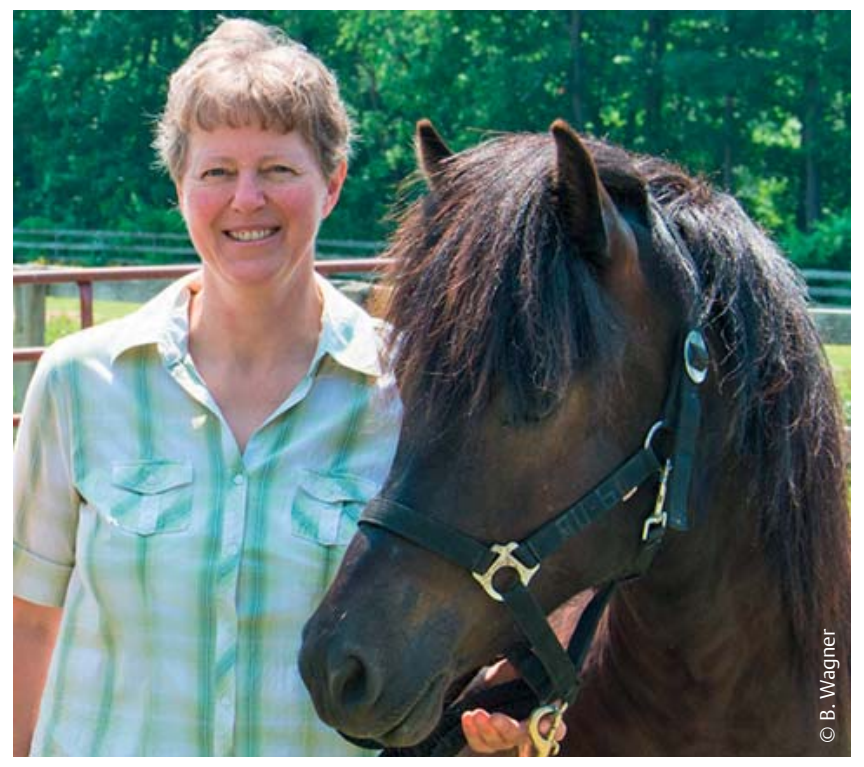

Abb. 1 Prof. Bettina Wagner mit einem Pferd, das an der Cornell-Sommerekzemstudie teilnimmt. Ziel dieser Studie ist es, die Entstehung des Sommerekzems tiefer zu verstehen sowie verbesserte Allergietests und Behandlungsmethoden zu entwickeln.

langer Zeit (Anfang der 1980er) durchgeführt wurde, in der eine Gruppe aus England gezeigt hat, dass bei dieser Erkrankung IgE-Antikörper in der bronchialen Lavage vorkommen. Trotz vieler Versuche, die Beteiligung von IgE in weiteren Studien zu zeigen, konnte dies danach nie wieder bestätigt werden. Wir sind heute nicht mehr sicher, ob die nachgewiesenen Antikörper in der ersten Studie aus England wirklich nur IgE-Antikörper waren. Die Spezifität des verwendeten Tests wird mittlerweile infrage gestellt. Aufgrund dieser ursprünglichen Publikation wurde immer wieder diskutiert, ob die COPD eine IgE-vermittelte Allergie ist. Zu diesem Zeitpunkt ist aber zumindest fraglich, ob es sich um eine IgE-vermittelte, allergische Erkrankung handelt. Wir glauben, dass die COPD in erster Linie eine Überreaktion des Respirationstrakts auf verschiedene Umweltkomponenten wie beispielsweise Schimmelpilze ist, und dass die Erkrankung in erster Linie eine inflammatorische Ätiologie hat, die nur in einigen wenigen Fällen mit einer IgE-vermittelten Komponente einhergeht.
Welche Allergene kommen bei den allergisch bedingten Erkrankungen infrage?

Fangen wir mit der Urtikaria an: Urtikaria wird typischerweise durch Nahrungsmittelallergene verursacht. Welche Allergene beteiligt sind, ist im Einzelfall oft sehr schwierig herauszufinden. Im Prinzip können alle Nahrungsmittelbestandteile Auslöser sein. Häufig versucht man, verschiedene Komponenten aus der Diät rauszunehmen und dadurch herauszufinden, worauf das Pferd reagiert. Bei manchen Pferden kann Urtikaria nur einmalig oder sehr sporadisch vorkommen. Es gibt aber auch Fälle, in denen das Problem immer wieder auftritt und extreme Ausmaße annehmen kann. Es ist schwierig festzustellen, welche Allergene beteiligt sind und die Allergietests helfen meist nicht weiter.

\section{Und beim Sommerekzem?}

Das Sommerekzem ist die am besten untersuchte allergische Erkrankung beim Pferd. Die Ätiologie und Immunologie sind weitgehend erforscht und die IgE-vermittelte, allergische Ursache der Erkrankung ist seit vielen Jahren bestätigt. Beim Sommerekzem ist mittlerweile bekannt, dass 
Allergene von Culicoides-Mücken für die Auslösung der Allergie verantwortlich sind. Auch andere Mücken-Spezies sind als Auslöser diskutiert worden, aber ein kausaler Zusammenhang wurde tatsächlich nur für Culicoides gezeigt. Wir wissen, dass sie zumindest die Hauptursache für diese Erkrankung sind.

Mittlerweile wurde eine ganze Reihe potenzieller Allergene aus dem Speichel der Culicoides-Mücken isoliert und beschrieben: Welche die Auslöser im Einzelfall sind, kann je nach Pferd variieren, und welche der Allergene die Allergie bei den meisten Pferden vorantreiben, wissen wir im Moment auch noch nicht. In den letzten 10 Jahren wurde in diesem Bereich sehr viel gearbeitet, um die auslösenden Allergene zu identifizieren, damit sie in naher Zukunft für verschiedene Tests und auch für Immuntherapie zur Verfügung stehen. Gegenwärtig arbeiten mehrere Forschungsgruppen daran, die Hauptallergene, die diese Erkrankung auslösen, zu identifizieren. Dies kann auch regional unterschiedlich sein und erfordert daher eine internationale, vergleichende Analyse.

Welche Möglichkeiten gibt es, allergische Erkrankungen labordiagnostisch nachzuweisen?

Auf dem Markt gibt es zurzeit mehrere diagnostische Möglichkeiten, die allgemein als „Allergietests“ bezeichnet werden und zum Ziel haben, das oder die auslösenden Allergene für das jeweils betroffene Pferd zu identifizieren. Der Goldstandard in der Allergie-Diagnostik ist der Intradermaltest. Dabei wird dem Pferd eine ganze Reihe von Allergenen in die Haut injiziert. Die Pferde antworten mit einer spontanen, lokalen Schwellung auf die entsprechenden Allergene. Ein Intradermaltest ist jedoch aufwendig, nicht immer gut toleriert vom Pferd und manchmal auch unerwünscht vom Besitzer, weil das Pferd vor der Allergeninjektion am Hals geschoren werden muss. Es gibt also Akzeptanzprobleme und dazu praktische Probleme, weil die Besitzer mit dem Pferd in die Klinik kommen müssen, da die meisten praktischen Tierärzte diesen Test nur in Ausnahmefällen durchführen. Man braucht ein gewisses Training, um den Test korrekt durchzuführen (die verschiedenen Allergene müssen zügig und trotzdem gleichmäßig injiziert werden). Oft werden 60 oder mehr Allergene gleichzeitig getestet, sodass der Test in der Praxis selten angeboten wird.

Meistens greifen praktische Tierärzte deshalb auf andere Tests zurück, oder starten die Behandlung aufgrund der klinischen Symptomatik ohne weitere Tests. Die Therapie bei Allergien ist zurzeit noch symptomatisch und hängt nur bedingt von den beteiligten Allergenen ab. Manchmal möchten Tierarzt und Besitzer jedoch herausfinden, welche Allergene ursächlich für die Erkrankung sind. Dies ist häufig erforderlich, um zu vermeiden, dass das Pferd erneut mit dem Allergen in Kontakt kommt, oder aber Tierarzt und Besitzer denken über eine Immuntherapie nach. Diese wird in der Humanmedizin angeboten und ist auch beim Pferd versucht worden. In diesen Fällen braucht man einen Nachweis des auslösenden Allergens - einen sogenannten Allergietest. Die Tests, die für das Pferd zur Verfügung stehen, sind entweder sogenannte ELISA oder aber Degranulations-Tests. Auf dem Markt gibt es verschiedene Tests mehrerer Anbieter.

Welche Probleme haben diese Tests? Alle Allergietests basieren auf dem Nachweis von entweder frei im Serum vorhandenen oder zell-gebundenen, allergenspezifischen IgE. Alle gegenwärtig verfügbaren Tests haben Einschränkungen.

Die erste Gruppe von Tests, die auf dem ELISA-Prinzip beruhen, versucht, IgE im Serum nachzuweisen. Das grundsätzliche Problem für den IgE-Nachweis im Serum ist die sehr geringe Konzentration der IgE-Antikörper (im Bereich von ng/ml bis $\mu \mathrm{g} / \mathrm{ml}$ im Serum). Im Gegensatz dazu ist die Konzentration von IgG-Antikörpern im Serum ca. 10000-fach höher. Auf der ELISA-Oberfläche kommt es dann zur Kompetition (Konkurrenz) zwischen den verschiedenen Antikörpern aus dem Serum. Da das Verhältnis von allergen-spezifischen $\operatorname{IgE}: \operatorname{IgG}$ ca. 1:10000 ist, binden nur sehr wenige IgE-Antikörper an die Testplatte. Dies bedingt, dass AllergieTests, die auf dem ELISA-Prinzip basieren, generell eine sehr geringe analytische Sensitivität haben. Ein Pferd muss also sehr viel allergen-spezifisches IgE haben, um in so einem Test positiv zu sein. Hochallergische Pferde sind oft positiv, aber häufig gegen sehr viele Allergene. Einige allergische Pferde können falsch-negativ sein. Manchmal reagieren nicht-allergische Pferde positiv in diesen Tests. All dies macht die Interpretation von Allergietests schwierig.

Und wie ist die Spezifität dieser Tests? Die Spezifität ist der andere Knackpunkt: Es gibt oft falsch-positive Reaktionen. Diese können sowohl durch Kreuzreaktivität mit anderen Allergenen oder auch durch Nachweisreagenzien entstehen, die nicht $100 \%$ IgE-spezifisch sind. Aus wissenschaftlicher Sicht ist es oft fraglich, ob ein bestimmter Test wirklich IgE-spezifisch ist, da eine genaue Beschreibung der Testreagenzien nur für sehr wenige Allergietests verfügbar ist. Insgesamt sind Allergietests, die auf den IgE-Nachweis beruhen, oft wenig zuverlässig. Es kommen sowohl falsch-positive als auch falsch-negative Ergebnisse vor. Häufig machen die Testergebnisse in der Praxis wenig Sinn und somit kann man nicht wirklich sagen, was sie bedeuten.

Welche anderen Tests werden angeboten? Eine zweite Gruppe von Tests, die angeboten wird, beruht auf dem Nachweis von zell-gebundenem IgE und zellulärer Reaktion. Einer dieser Tests kommt aus Hannover und ist mittlerweile als FIT (Funktioneller-In-vitro-Test) bekannt. Es handelt sich um einen Degranulationstest: Er misst die Menge des Histamins, das nach Allergenkontakt von bestimmten Blutzellen, den sogenannten Basophilen, freigesetzt wird. Der Test wird mit Erfolg für Culicoides-Allergen bei der Sommerekzemdiagnostik eingesetzt, hat aber ein begrenztes Allergenspektrum und ist nicht für viele andere Allergene (z.B. Gräser, Kräuter, Bäume usw.) verwendbar. Eine Alternative dazu ist der Nachweis von Leukotrienen, einer anderen Gruppe von Mediatoren, die von Basophilen produziert werden. Der CAST-Test basiert auf dem Nachweis von Leukotrienen und benutzt verschiedene Insektenallergene.

In welchen Fällen empfehlen Sie, diese Tests durchzuführen?

Für die meisten Allergietests gibt es keine ausreichenden wissenschaftlichen Vali- 
dierungsdaten über die Testgenauigkeit. Vergleiche zwischen verschiedenen kommerziellen Allergietests weisen meist nur geringe Übereinstimmung auf. Zusammengefasst bedeutet dies, dass die Ergebnisse dieser Tests nicht wirklich das oder die Allergene identifizieren, die für die klinische Erkrankung des Pferdes verantwortlich sind. Einige wissenschaftliche Publikationen haben gezeigt, dass viele Tests allergische Pferde nicht von nicht-allergischen unterscheiden können. Solange Validierungsdaten nicht verfügbar sind, gibt es aus wissenschaftlicher Sicht keine Empfehlung für diese Tests. Auf Grundlage der vorhandenen wissenschaftlichen Literatur sind Tests, die die zelluläre Sensibilisierung nachweisen - wie der Intrakutantest oder die zellulären Degranulationstests (FIT und CAST) - besser zum Allergennachweis geeignet als ELISATests, die auf dem Nachweis von SerumIgE beruhen. Insgesamt ist im Bereich der Allergietests noch Einiges an Forschung und Verbesserung erforderlich.

Besonders kritisch sollten Allergietests betrachtet werden, wenn die Ergebnisse für eine Immuntherapie (Hypo- oder Desensibilisierung) verwendet werden sollen. Es gibt zurzeit keinen ausreichenden Nachweis oder wissenschaftliche Studien, die zeigen, dass eine Hyposensibilisierung, die auf den Ergebnissen dieser Tests basiert, bei den Pferden wirklich wirkt. Vieles wird aus der Humanmedizin übertragen, aber der Erfolg beim Pferd ist tatsächlich noch nicht bewiesen. Das Problem ist, dass man nicht mit Sicherheit sagen kann, gegen welche Allergene das Pferd tatsächlich allergisch ist. Solange Allergietests keine eindeutigen Ergebnisse geben, ist eine Immuntherapie nicht die richtige Lösung.

Welche therapeutischen Möglichkeiten stehen dem Tierarzt zur Verfügung?

Die Therapie bei Allergien ist symptomatisch und besteht in erster Linie aus der Allergenvermeidung. Bei Culicoides-Allergien bedeutet es z.B., dass das Pferd im Sommer nicht den ganzen Tag auf der Weide laufen kann und kontinuierlich Decken und Fliegenschutz verwendet werden müssen. Um eine Culicoides-Exposi- tion zu vermeiden, muss dies konsequent, also täglich, den ganzen Sommer über durchgeführt werden. Dies ist ein komplexes Managementproblem, immer aufwendig und manchmal nicht möglich.

Die Alternative ist eine symptomatische Therapie mit Kortikosteroiden. Wenn diese als Langzeitbehandlung den ganzen Sommer über durchgeführt wird, ist das Pferd monatelang in einem immunsupprimierten Zustand, was viele Nebenwirkungen haben kann. Wenn die Allergie-Symptome sehr stark sind - z.B. bei Urtikaria oder bestimmten Phasen des Sommerekzems - kann diese Therapie durchaus helfen und die Symptome kurzfristig lindern. Andere (symptomatische) Therapien beruhen auf der Gabe von Antihistaminika. Beim Sommerekzem ist auch eine regelmäßige lokale Behandlung der betroffenen Hautbereiche hilfreich, z.B. Wunden sauber halten oder Präparate auftragen, die Fliegen abhalten und die Wundheilung verbessern.

Gibt es also keine ätiologische Therapie? Nein, zurzeit gibt es keine gesicherte ätiologische Therapie für Allergie bei Pferden. Die einzige Maßnahme, die beim Sommerekzem wirklich helfen kann, ist die Allergenvermeidung. Die Haltung des Pferdes muss so verändert werden, dass der Kontakt mit den Culicoides-Mücken stark eingeschränkt wird. Es ist vergleichbar, als ob man eine Katzenallergie hätte und gleichzeitig Katzen in der Wohnung halten würde. Wenn die Katzen nicht mehr da sind, ist auch das auslösende Allergen nicht mehr vorhanden und man kann eine deutliche Verbesserung erreichen.

Für eine alternative, verlässliche Therapie ist viel mehr Forschung nötig. Man kann nur hoffen, dass wir in den nächsten 10 Jahren etwas mehr in diesem Bereich herausfinden.

Was halten Sie dann von der Desensibilisierung?

Die Desensibilisierung/Immuntherapie ist der richtige Ansatz. Beispiele aus der Humanmedizin zeigen, dass damit vielen Pa- tienten geholfen werden kann. Beim Pferd sind wir aber zurzeit noch nicht in der Lage, mit einer ausreichenden Sicherheit zu sagen, gegen welche Allergene von Culicoides Pferde eigentlich allergisch sind und wie die Immuntherapie für das einzelne Pferd aussehen müsste. Darüber hinaus sind viele Fragen wie Allergenmenge und Häufigkeit der Verabreichung noch ungeklärt. Aus der gegenwärtigen Sicht kann man die Desensibilisierung beim Pferd also nur als ein „Experiment“ bezeichnen. Es ist mehr als verständlich, dass Besitzer etwas für ihr krankes Pferd tun wollen, um den dauerhaften Juckreiz und die klinischen Symptome zu verbessern. Jedoch ist das Risiko sehr hoch, dass man viel Geld bezahlt ohne etwas zu erreichen oder aber die Situation mit einer solchen „Therapie“ sogar noch verschlimmert.

Liegt bei Allergien eine genetische Komponente zugrunde?

Eine Allergie ist eine sogenannte multigenetische bzw. multifaktorielle Erkrankung. Mehrere Gene sind beteiligt und man kann die verantwortlichen Gene nicht einfach identifizieren oder aus der Pferdepopulation durch Zucht eliminieren. Außerdem gibt es neben den genetischen Komponenten auch noch Umweltkomponenten, die eine mindestens ebenso wichtige Rolle spielen. Es ist insofern extrem schwierig vorherzusagen, ob ein Pferd allergisch wird oder nicht. Die Antwort ist also ja: Es gibt eine genetische Komponente, aber sie hilft uns nicht, das Problem zu beheben oder früher zu erkennen.

Sollten betroffene Tiere von der Zucht ausgeschlossen werden?

Eher nicht. Die Umweltkomponenten (z.B. wann die Pferde zuerst in Kontakt mit Allergenen kommen) beeinflussen die Ausprägung der klinischen Allergie deutlich mehr als die genetische Veranlagung. Allergische Tiere von der Zucht auszuschließen wird das Problem der Allergie nicht beheben.

\section{Online}

http://dx.doi.org/10.1055/s-0041-110021 